\title{
Towards a phylogenetic analysis of galaxy evolution: a case study with the dwarf galaxies of the Local Group ${ }^{\star}$
}

\author{
D. Fraix-Burnet ${ }^{1}$, P. Choler ${ }^{2}$, and E. J. P. Douzery ${ }^{3}$ \\ 1 Laboratoire d'Astrophysique de Grenoble, BP 53, 38041 Grenoble Cedex 9, France \\ e-mail: fraix@obs.ujf-grenoble.fr \\ 2 Laboratoire d'Écologie Alpine, BP 53, 38041 Grenoble cedex 9, France \\ ${ }^{3}$ Laboratoire de Paléontologie, Phylogénie et Paléobiologie, Institut des Sciences de l'Évolution de Montpellier, \\ 34095 Montpellier Cedex 5, France
}

Received 27 February 2006 / Accepted 25 April 2006

\section{ABSTRACT}

\begin{abstract}
Context. The Hubble tuning-fork diagram has always been the preferred scheme for classifying galaxies. It is based only on morphology. In contrast, biologists have long taken the genealogical relatedness of living entities into account for classification purposes. Aims. Assuming branching evolution of galaxies as a "descent with modification", we show here that the concepts and tools of phylogenetic systematics that are widely used in biology can be heuristically transposed to the case of galaxies.

Methods. This approach, which we call "astrocladistics", is applied to dwarf galaxies of the Local Group and provides the first evolutionary tree for real galaxies.

Results. The trees that we present here are solid enough to support the existence of a hierarchical organisation in the diversity of dwarf galaxies of the Local Group. They also show that these galaxies all stem from a common ancestral kind of object. We find that some kinds of dIrrs are progenitors of both $\mathrm{dSphs}$ and other kinds of dIrrs. We also identify three evolutionary groups, each one with its own characteristics and own evolution.

Conclusions. The present work opens a new way to analysing galaxy evolution and a path towards a new systematics of galaxies. Work on other galaxies in the Universe is in progress.
\end{abstract}

Key words. galaxies: fundamental parameters - Galaxy: evolution - galaxies: formation

\section{Introduction}

Since Hubble (1922, 1926), classification of galaxies relies largely on morphology: spirals (flattened galaxies with spiral arms), barred spirals, ellipticals (galaxies with no obvious pattern), and irregulars (Kormendy \& Bender 1996; Roberts \& Haynes 1994). Subdivisions have been created since then (de Vaucouleurs 1959; Sandage 1961) in an attempt to transform morphology into a more quantitative parameter. The use of a limited number of additional properties - radio and X-ray properties, environment, nucleus activity, gas content, star formation, colours, and others - has led to the recognition of numerous classes that are essentially catalogues of objects. This traditional approach to classification might look inadequate for describing the now acknowledged complexity of galaxies. Multivariate analysis of these properties has been once proposed to tackle the problem of synthesizing the classificatory information included in independent characteristics (Whitmore 1984; Watanabe et al. 1985). However, these approaches are still based on estimations of the general similarities.

In fact, galaxies are complex systems in which several physical and chemical processes govern the evolution of their basic constituents: stars, gas, dust, molecules, and probably black holes (e.g. Vilchez et al. 2001). Surprisingly, besides Hubble's

^ Figure 3 is only available in electronic form at http://www.edpsciences.org primer hypotheses of the evolution of galaxies leading to the famous Hubble or tuning-fork diagram (Hubble 1936), there has been no further attempt to base classification of galaxies on historical/evolutionary relationships. Nowadays, the physical and chemical processes are often roughly understood individually and sometimes even modelled (e.g. Sauvage et al. 2002); but they are not collectively integrated, so that grasping the causes of galaxy diversity in their entire complexity remains a difficult task.

Similar concerns have already been addressed in the study of biological diversity. Nearly 150 years ago, Darwin (1859) suggested that the hierarchical classification of living organisms should reflect their genealogical relationships. This was an incredibly successful idea that drove biological systematics into a new era. There is now a common agreement that a natural classification should be derived from phylogenetic trees, i.e. a branching structure describing the evolutionary relationships of a set of biological entities or taxa (Wiley et al. 1991). Accordingly, the concepts and methods of phylogenetic systematics have been successfully applied to other sets of entities for which historical or evolutionary relationships could be documented; examples are found in linguistics (Wells 1987) and stemmatics (Robinson \& Robert 1996).

We therefore hypothesize that galaxies can be classified in a natural hierarchy of nested groups reflecting evolution. Our purpose is thus to introduce the parameter "time" in the 
classification scheme, using all available and suitable descriptive characters ${ }^{1}$, and to propose an integrated way of representing galaxy diversity. We have named this approach "astrocladistics" (Fraix-Burnet et al. 2003; Fraix-Burnet 2004). The method and associated concepts are presented in detail elsewhere, together with an analysis of two samples of simulated galaxies (Fraix-Burnet et al. 2006a,b). In this paper, we present the first application of astrocladistics to real galaxies. We chose to focus on the Dwarf galaxies of the Local Group for which a reasonable amount of complete and homogeneous data are available. According to the "hierarchical scenario"2 of galaxy formation, these galaxies could belong to small dark-matter halos that are the building blocks of larger structures that may host big galaxies. In this respect, dwarf galaxies are not considered as being formed by the merging of smaller structures; but because of the relatively shallow gravitational well, they are certainly subject to disturbances and sweeping. However, this hierarchical scenario of galaxy formation has some difficulties, like predicting too many small structures that are not seen in the form of dwarf galaxies (e.g. Moore et al. 1999; Venn et al. 2004). The Local Group could also represent an evolutionary microcosm in which the environments for all the dwarfs have been somewhat similar. Here we scored a variety of characters, and inferred the most parsimonious history that describes the evolution of dwarf galaxies of the Local Group.

\section{Methods}

In this section, we first outline briefly some concepts of astrocladistics, then describe the data used for our analysis, and finally present the process of tree construction. All details on astrocladistics are given in Fraix-Burnet et al. (2006a, 2006b).

\subsection{Conceptual framework}

A cornerstone of phylogenetic systematics is to focus on derived character states to infer common ancestry relationships (Hennig 1965). Such characters may be viewed as evolutionary novelties appearing in a particular lineage. It is assumed that two closely relative objects (also called taxa because they can be individuals, groups, or species) share derived characters, which presumably originate in their common ancestor. Regarding classification, phylogenetic systematics defines clades as evolutionary groupings that are significant for evolution by containing all offspring from a common ancestor, and their members share common derived states, that is to say, a common history.

Now we consider two identical galaxies hence that belongs to the same class. If they evolve independently and are isolated, the number of processes at work within each galaxy makes it probable that after a given time, they will be different enough to be put into two different classes. For instance, if they both had a spiral shape, they would remain this way, but one could have developed a bar or had a starburst activity modifying its stellar population. In addition, galaxies live in an environment made of intergalactic gas and gravitational potential fashioned by dark matter and other galaxies (Sauvage et al. 2002). If two galaxies interact with each other, the dynamics will usually be affected differently in both because of asymmetries in the encounter, so

\footnotetext{
${ }^{1}$ In cladistics, a character is a trait, a descriptor, an observable, or a property, that can be given at least two states characterizing the evolutionary stages of the object for that character.

2 This is not to be confused with the hierarchical organisation of galaxy diversity dealt with in this paper.
}

that they could then be placed in two separate classes. In all cases, one class gives birth to at least two classes. This is the definition of branching evolution. Its driver is mainly the randomness of external interactions and to a lesser extent that of internal processes. This branching evolution results in a hierarchical or tree-like organisation of the diversity.

\subsection{Data}

We used data from Mateo (1998) on 36 galaxies and identified 25 characters. For each character, we discretized the whole range of values in up to 8 bins, plus the unknown value "?"3. These are assumed to be evolutionary states. For instance, if we accept that metallicity increases with time, then a very low value would be an ancestral state, whereas a high value would be a derived state. This has to be adapted to the sample under study. We imposed 15 characters to be ordered (see Table 1), i.e. changes between two adjacent states are more probable than between distant ones, independent of the direction of the change. The morphological character (dIrr, Intermediate, and dSph) was discarded in the analysis because it differs notably from the other ones by being the only qualitative property and also being somewhat subjectively defined. It was coded into three states merely for projection onto the result trees. We thus ended up with a matrix of 36 objects described by 24 characters, the total percentage of unknown values being $33 \%$ ).

The outgroup, used to root the tree - i.e., to orientate the evolutionary processes - indicates the ancestral states of the characters for which transformation can be hypothesized within a reasonably accepted paradigm. Given the complicated evolutionary behaviour of Dwarf galaxies, for this first astrocladistics analysis, we decided to choose a galaxy in the sample. The advantage is that the result tree can be easily rerooted with any of the sample objects depending on one's own guess, because the structure of the tree does not change. The SagDIG galaxy is chosen here as root because it has the highest ratio $\mathrm{MHI} / \mathrm{M}$ of the sample. It is interesting to note that SagDIG seems to be at the edge of our Local Group (van den Bergh 2000).

\subsection{Tree construction}

An important aspect of phylogenetic systematics is to avoid grouping objects based on similarities due to evolutionary convergences or reversals. In phylogenetic analysis, maximum parsimony (Fitch 1971) is one optimality criterion for selecting among competing trees. It states that the most probable evolutionary scenario is the simplest one as measured by the total number of character state changes (called steps). The most parsimonious tree thus corresponds to the simplest evolution scenario that is compatible with all the input data. The use of the parsimony principle also minimises the number of convergences or reversals. We used maximum parsimony to identify the tree best for representing our data matrix. Since there is an important number of unknown values in the data, the full tree obtained with the 36 galaxies is not extremely robust in the sense that slightly different trees could also fit the data. To assess its significance, we thus looked for smaller trees and kept the more robust one we could find keeping the subsample as large as possible. Evaluating the reliability of tree nodes was done through bootstrap (percentages of node occurence obtained after 1000 resampling of characters, Felsenstein 1985) and decay (minimum

\footnotetext{
3 Tables are available on http://hal .ccsd.cnrs/aut/fraix-burnet
} 
Table 1. List of characters used in the cladistics analysis. The constraint on the evolution of each character is indicated by "o" for "ordered" and "u" for "unordered".

\begin{tabular}{rlc}
\hline \hline & Character & Constraint \\
\hline 1 & Ellipticity & $\mathrm{u}$ \\
2 & Core radius & $\mathrm{o}$ \\
3 & $V$ Luminosity (integrated) & $\mathrm{o}$ \\
4 & $B-V$ (integrated) & $\mathrm{o}$ \\
5 & $U-B$ (integrated) & $\mathrm{o}$ \\
6 & Total mass & $\mathrm{o}$ \\
7 & Central mass density & $\mathrm{u}$ \\
8 & HI mass & $\mathrm{o}$ \\
9 & Mass / luminosity ratio & $\mathrm{o}$ \\
10 & HI mass / total mass ratio & $\mathrm{o}$ \\
11 & HI mass / B luminosity ratio & $\mathrm{o}$ \\
12 & Dust mass & $\mathrm{o}$ \\
13 & HI flux & $\mathrm{o}$ \\
14 & CO flux & $\mathrm{u}$ \\
15 & H $\alpha$ flux & $\mathrm{o}$ \\
16 & Fe / H ratio & $\mathrm{o}$ \\
17 & O / H ratio & $\mathrm{u}$ \\
18 & N / O ratio & $\mathrm{o}$ \\
19 & Star Formation Rate & $\mathrm{u}$ \\
20 & Rotational velocity & $\mathrm{u}$ \\
21 & Velocity dispersion of ISM & $\mathrm{u}$ \\
22 & Rotational velocity / Velocity dispersion & $\mathrm{o}$ \\
23 & Maximum rotational velocity & $\mathrm{u}$ \\
24 & Central velocity dispersion & $\mathrm{u}$ \\
\hline & &
\end{tabular}

number of character-state changes to be added to tree length to break the corresponding node, Bremer 1994) estimates. All calculations were conducted with PAUP*4.0b10 (Swofford 1998) and the tree interpretation was done with Mesquite (Maddison $\&$ Maddison 2004).

\section{Results}

We found a fully resolved tree with 14 galaxies (Fig. 1). It has a total of 167 steps (character-state changes), while bootstrap and decay values are high for nearly all nodes (Fig. 1), indicating that most tree nodes are strongly supported by the data. No better trees could be found with 14 or more objects. Regarding the groupings and evolutionary behaviours of characters, that is, the resulting phylogeny, all robust trees we found with 14 or slightly less galaxies are all compatible, as they are with the full tree discussed below.

Performing an analysis with the full sample of 36 galaxies, we found 1041 most parsimonious trees, each having 317 steps. The strict consensus of all of them is shown in Fig. 2. It is remarkably well resolved despite the large number of unknown values. The strict consensus means that the nodes present on the tree are found in all 1041 individual trees. We must warn that this tree is not as robust for the bootstrap and decay estimators as the previous one so that its very detailed structure should be used with some caution. However, the two trees are fully compatible and the character behaviours, as discussed below, are identical, pointing toward the same evolutionary history of diversification.

Generally speaking, the farther a galaxy is from the root (SagDIG), the more it is differentiated from it. It is not necessarily more evolved since it depends on the timescale of each evolutionary process (see 2006a,b for a more complete discussion). For simplification at the level of the discussion and because we lack an evolutionary clock, we here identify diversification and evolution. The very existence of resolved and robust trees

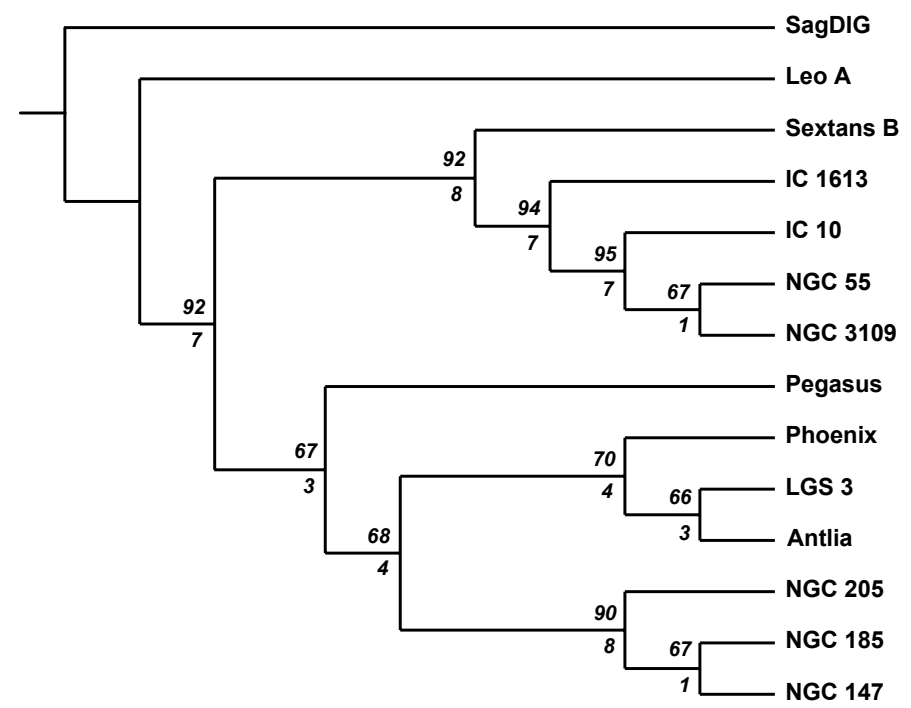

Fig. 1. Best tree with 14 galaxies obtained by a maximum parsimony analysis of 24 characters as described in the text. It has 167 steps, the consistency index $=0.68$, retention index $=0.75$, rescaled consistency index $=0.51$. Numbers at each node are bootstrap (above) and decay (below) values. Bootstrap percentages are obtained after 1000 resamplings of the characters. Decay indexes correspond to the minimum number of character-state changes to be added to the tree length to break the corresponding node.

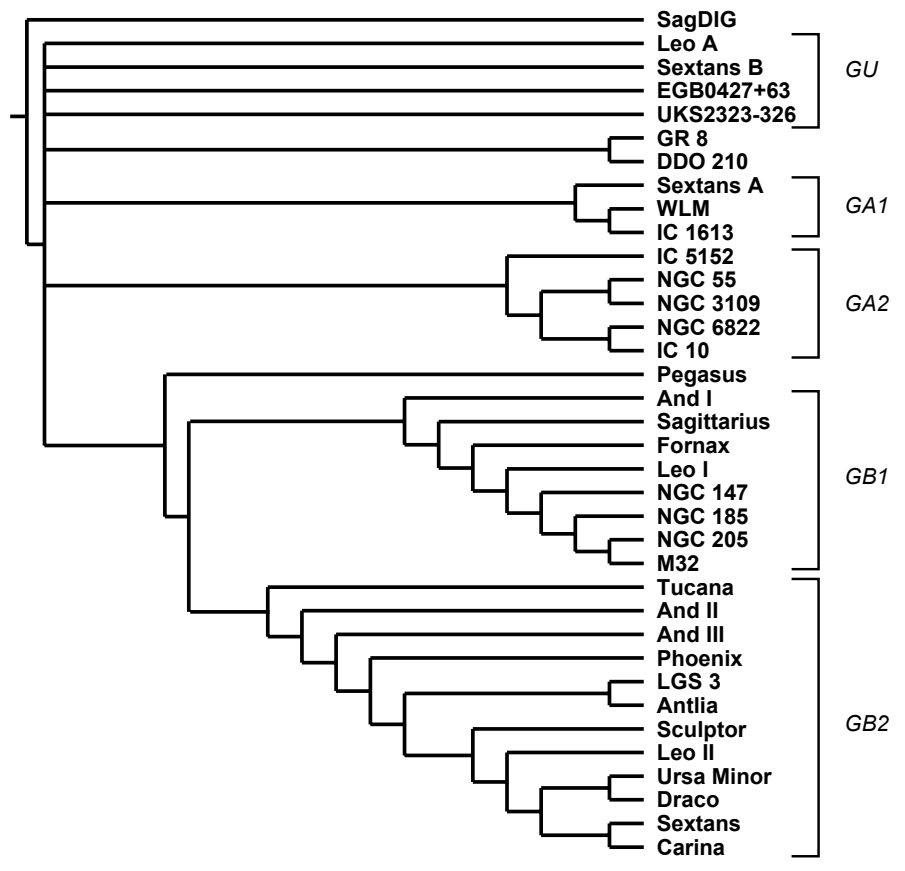

Fig. 2. This tree with the 36 dwarf galaxies of the Local Group is the strict consensus of those 1041 most parsimonious trees that have 317 steps each. Groups corresponding to sub-structures are indicated and discussed in the text.

implies that all these galaxies share a common ancestor with respect to SagDIG; that is, they all stem from the same kind of objects. In addition, diversity in the Dwarf galaxies of the Local Group arranges itself in a hierarchical way.

Five groups are identified in Fig. 2 and defined in Table 2. In this table, starred names indicate galaxies present on the small tree in Fig. 1. The unique characteristics belong only to the group, while the other characteristics are found in other groups. 
Table 2. Characteristics of groups identified in Fig. 2.

\begin{tabular}{|c|c|c|c|}
\hline Group & Members & Unique characteristics & Other characteristics \\
\hline GU & $\begin{array}{l}\text { Leo A * } \\
\text { Sextans B * } \\
\text { EGB0427+63 } \\
\text { UKS2323-326 }\end{array}$ & & $\begin{array}{l}\text { High MHI/BLum } \\
\text { (dIrr) }\end{array}$ \\
\hline GA1 & $\begin{array}{l}\text { Sextans A } \\
\text { WLM } \\
\text { IC } 1613 *\end{array}$ & & $\begin{array}{l}\text { Low Central Mass density } \\
\text { Low central M/L } \\
\text { High MHI/BLum } \\
\text { (dIrr) }\end{array}$ \\
\hline GA2 & $\begin{array}{l}\text { IC } 5152 \\
\text { NGC } 55 * \\
\text { NGC } 3109 * \\
\text { NGC } 6822 \\
\text { IC } 10 *\end{array}$ & $\begin{array}{l}\text { High Mass } \\
\text { High HI Mass } \\
\text { High Dust Mass } \\
\text { High HI Flux } \\
\text { High CO Flux } \\
\text { High H } \alpha \text { Flux } \\
\text { High SFR } \\
\text { High Vrot/Vdisp }\end{array}$ & $\begin{array}{l}\text { High } V \text { Luminosity } \\
\text { Low Central Mass density } \\
\text { Low central M/L } \\
\text { High MHI/BLum } \\
\text { (dIrr) }\end{array}$ \\
\hline GB1 & $\begin{array}{l}\text { And I } \\
\text { Sagittarius } \\
\text { Fornax } \\
\text { Leo I } \\
\text { NGC } 147 * \\
\text { NGC } 185 * \\
\text { NGC } 205 * \\
\text { M32 }\end{array}$ & $\begin{array}{l}\text { High U-B } \\
\text { Highly increasing Central Mass density } \\
\text { Decreasing central M/L } \\
\text { Low MHI/BLum }\end{array}$ & $\begin{array}{l}\text { Increasing } V \text { Luminosity } \\
\text { Increasing } B-V \\
\text { Very low HI Mass } \\
\text { Very low MHI/M } \\
\text { (dSph) }\end{array}$ \\
\hline GB2 & $\begin{array}{l}\text { Tucana } \\
\text { And II } \\
\text { And III } \\
\text { Phoenix * } \\
\text { LGS } 3 * \\
\text { Antlia * } \\
\text { Sculptor } \\
\text { Leo II } \\
\text { Ursa Minor } \\
\text { Draco } \\
\text { Sextans } \\
\text { Carina }\end{array}$ & $\begin{array}{l}\text { Low and decreasing V Luminosity } \\
\text { Increasing central M/L } \\
\text { Decreasing MHI/BLum } \\
\text { Low } \mathrm{Fe} / \mathrm{H}\end{array}$ & $\begin{array}{l}\text { Increasing } B-V \\
\text { Increasing Central Mass density } \\
\text { Very low HI Mass } \\
\text { Very low MHI/M } \\
\text { (dSph) }\end{array}$ \\
\hline
\end{tabular}

The words "increasing" or "decreasing" refer to evolution within the group as oriented on the tree rooted with the SagDIG galaxy. The morphology (in parentheses) was not used to derive the tree. These groups correspond to structures on the tree, mainly lineages, except for $G U$, which gathers a set of unresolved branches for convenience in the discussion. After SagDIG, the root of the tree, there are four groups with unresolved respective phylogenies, $G U, G A 1, G A 2$, and $G B$ (GB1 and $G B 2$ ), plus the pair GR 8-DDO 210. In contrast, the branch after Pegasus diverges in two clear subtrees, $G B 1$ and $G B 2$, which are the most diversified from SagDIG among the whole sample. There are three pairs within GB2: LGS 3-Antlia, Ursa Minor-Draco, and Sextans-Carina. On the small tree (Fig. 1), the phylogeny is fully resolved, and the above groups are present with at least one object representative of each (indicated in Table 2). After the branch toward Leo A, there are only two diverging branches, one leading to $G A 1$ then to $G A 2$, the other one finally leading to another binary bifurcation toward $G B 1$ and $G B 2$. Note that $G A$ (1 and 2) and $G B 2$ have very high bootstrap and decay values, indicating that they probably constitute a true evolutionary group. The support for $G B 1$ is somewhat less but still very significant. In other words, the small tree of Fig. 1 is a phylogeny of the groups defined in Table 2.

The reasons for the tree structure lies in the evolution of the characters. Their values are projected onto the larger tree in
Fig. 3 in order to visualize both the character evolution and the group properties. The latter are summarised in Table 2. It appears that, except for $G U$, which by construction cannot be considered to form an evolutionary group because the relative placement of galaxies in $G U$ is unknown, and groups can be unambiguously characterised by a set of properties. The three groups $G A 2, G B 1$, and $G B 2$ have unique characteristics. Group $G A 1$ resembles $G A 2$ but lacks a high $V$ luminosity. Following the small tree in Fig. 1, these two groups are related, implying an increase in $V$ luminosity with evolution along this branch. It is difficult to use the present data to conclude whether there are one or two different lineages.

On the contrary, there are undoubtedly two species identified by the two groups $G B 1$ and $G B 2$. In the first case, galaxies are specifically bright in $V$ and have a high central mass density but a low central ratio $\mathrm{M} / \mathrm{L}$, together with a low $\mathrm{MHI} / \mathrm{Blum}$. In the second case, galaxies are of low $V$ luminosity with a high and increasing (along this branch) central ratio $\mathrm{M} / \mathrm{L}$, a decreasing $\mathrm{MHI} /$ Blum, and may be more importantly a very low metallicity $(\mathrm{Fe} / \mathrm{H})$.

Assuming that SagDIG, as the root of the tree, resembles the common ancestor to Dwarf galaxies of the Local Group, a few characters seem to evolve regularly with the evolutionary scenario revealed by our tree: colours $(B-V$ and $U-B)$ might globally increase, while MHI/M, the HI mass, MHI/BLum might 
decrease. But the structure of the tree is not very regular, and most characters, like the $V$ luminosity, the total mass, or the dust mass, have different behaviours depending on the branches. Hence, the tree structure is explained by multiple parameters together with possibly a very few global evolutionary trends. The seemingly complex history of diversity in this sample might be due to the particular nature of dwarf galaxies, which shows that the different evolutionary stages cannot be established with only one or two descriptors.

This statement could seem to be contradicted by the morphology behaviour, as can be seen on the corresponding projection in Fig. 3. Despite this character not being used to build the tree, a perfect dichotomy appears between galaxies before Pegasus (dIrrs with groups $G U, G A 1$, and GA2) and after it (dSphs with groups GB1 and GB2). This galaxy is of the intermediate type. Interestingly, our 14-galaxy tree (Fig. 1) diverges after Pegasus into two groups, one of which is composed exclusively with three other intermediate-class galaxies (Antlia, LGS 3, Phoenix). But it corresponds to GB2 (Fig. 2), which also have dSphs. The last intermediate-type galaxy (DDO 210) is paired with GR 8 among the dIrrs. As clearly demonstrated by the structure of the tree, the dichotomy dSph-dIrr is far too simplistic to represent the diversity of dwarf galaxies, even solely in the Local Group, and misses all groups identified in this paper that are based on the information included in 24 characters. Indeed, spheroidal galaxies are all gathered in the group $G B$. These galaxies constitute a separate evolutionary lineages, and Fig. 1 stipulates more precisely that they were developed from ancestors of irregular morphologies. However, this is not enough for depicting the diversity and evolutionary history of these galaxies since there are probably at least two kinds of irregulars and certainly two rather different kinds of spheroidals. The character projections (Fig. 3) illustrate the well-known fact that the spheroidal dwarf galaxies have lost their HI gas, but goes further in describing the several kinds of evolutionary groups identified above in more detail.

\section{Discussion}

The trees show a hierarchical organisation of the dwarf galaxies of the Local Group with well-identified evolutionary groups. This result is particularly remarkable since these galaxies evolve in the same microcosm, which probably means less diversity. But more importantly, the two main hypotheses of astrocladistics, a common ancestor and a hierarchical organisation of diversity, are thus verified. The present result strongly stimulates the use of this approach to other galaxies, which is currently in progress.

The existence of a hierarchy in galaxy diversity makes it possible to track ancestral types of galaxies. Our result shows that the Dwarf galaxies of the Local Group very likely have a common ancestor, meaning that they derive from the same kind of objects. This seems to contradict Ricotti \& Gnedin (2005) who assume two different initial formation processes for irregulars and spheroidals. Even though SagDIG is here supposed to resemble the common ancestor of our sample, it is impossible to tell how much it resembles the common ancestor of all dwarf galaxies. Study of many more objects is needed. However, our result might not be incompatible with Ricotti \& Gnedin's result since it depends on the definition of both ancestor object and formation concept (see Fraix-Burnet et al. 2006a,b). In addition, dwarfs are currently thought to form within small dark-matter structures, but this formation process is far from being understood or even definitively established.
Assuming different stellar-formation histories, Ricotti \& Gnedin define three groups: survivors, true and polluted fossils. Only the true fossils correspond relatively well to one of our groups, GB2, except for Carina, LGS 3, and Leo II that they classify as polluted fossils. But our classification is based on other descriptors than just the stellar population. In the same manner, Grebel (2005) presents a diagram tentatively summarising the history of dwarf spheroidals based on star-formation episodes. If we compare the exemplar galaxies of her categories with our tree, we find that Draco ("old") and Carina ("episodic") both belong to GB2, while Fornax and Phoenix (both "young") belong respectively to $G B 1$ and $G B 2$, and Leo I ("intermediate") is close to Fornax. Again, the two classifications do not match for the same reason as above, indicating that the star-formation history is certainly insufficient for depicting the full diversity of the dwarf galaxies. In contrast, our data may be inadequate for characterising the stellar population in detail. Since an astrocladistics analysis reflects the knowledge at a given epoch and is thus never finished, extension of the present work in the future will have to include more descriptors as they become available for most of the galaxies.

Because the cladogram recapitulates evolutionary information for several characters, we think it could give a better insight on possible caveats of wordings like "young galaxy" and "old galaxy". All the galaxies of the sample are contemporaneous, but some resemble the ancestor more than others; that is, they look like "old" species. They are not necessarily "old" objects. Likewise, galaxies at the bottom of the tree are more diversified, but cannot be said to be "younger". For instance, Ursa Minor is often believed to have evolved little (Mateo 1998; Carrera et al. 2002; Mighell 1999) because it has old stars. In contrast, IC 10 have had strong and recent starburst activity (Mateo 1998; Wilcots \& Miller 1998). From our cladistic analysis, it would be meaningless to qualify one "younger" than the other; instead Ursa Minor seems to be more diversified from the ancestor as compared to IC 10. They do belong to two different species, each with its own evolutionary pathway, which diverged sometime in the history of these galaxies and of all their progenitors (see Fraix-Burnet et al. 2006a,b). Hence, we think it is tricky to speak of "old" or "young" galaxies based on star-formation history alone. It is preferable to refer to the stage of diversification.

The evolution of dwarf galaxies of the Local Group is often reduced to the segregation between dIrrs and dSphs (see e.g. Mateo 1998). For instance, one explanation for the depletion of HI gas in dSphs could be the proximity of a big galaxy. In Fig 3, each galaxy name has a colour corresponding to the sub-group to which it belongs (Mateo 1998). All members of the Milky Way family are spheroidals, but they do not belong to the same evolutionary group. There is no clear correlation with distance to the Milky Way. For the M31 (Andromeda) family, the four closest satellites (M32, NGC 205, NGC 147, and NGC 185) are grouped together in $G B 1$ with, among some others, Sagittarius, a very close satellite of our Milky Way. The other members are spread all over the tree with EGC0427+63 and IC 10 in $G U$ and GA2, respectively. The NGC 3109 family is spread over the tree, while the Local Group cloud members are spread over $G U$, $G A 1, G A 2$, and even GB2 for Tucana. Note that SagDIG belongs to this last family, but is certainly the farthest member of the Local Group since on its edge (van den Bergh 2000). Our conclusion is that the measured distance to a big galaxy is not enough to explain the diversity of the dwarf galaxies of the Local Group, even though nearly all companions of the two big galaxies of our Local Group belong to the groups $G B 1$ and $G B 2$, together with only three more "isolated" objects. One must keep 
in mind that the measured distance is the current distance, while the properties of galaxies are the result of a long history along a probably complicated trajectory that saw this distance change considerably.

Another example is given by conclusions like dIrrs being unlikely progenitors of dSphs (e.g. Grebel 2005). This kind of statement ignores the complexity of galaxies and their evolution. Comparing the two morphological types globally cannot be successful due to the wide variety of objects within each class (see e.g. Mateo 1998). The tree in Fig. 2 and the character projections (Fig. 3) illustrates that the evolutionary stage of a galaxy cannot be reasonably assessed with the sole morphology criterion. Our result instead shows that some kinds of dIrrs are progenitors of both $\mathrm{dSphs}$ and other kinds of dIrrs that diverged and developed their own lineage.

Our choice of SagDIG as root provides a globally satisfactory evolutionary scenario. This choice could easily be changed without modifying the structure of the tree, but then the interpretation would be different. Regularity of evolution was imposed on some characters (Sect. 2.2, Table 1), but this constraint does not imply monotonic behaviours as can be seen on the tree (Fig. 3, Sect. 3). More important, characters can evolve differently in different lineages. For instance, the $V$ luminosity regularly increases in $G B 1$, while it decreases in $G B 2$, making a global evolutionary behaviour quite complex and of low significance.

We have identified at least three evolutionary groups (Table 2). The first one (GA2) is composed of massive galaxies with a high star-formation rate, a lot of HI gas and dust, high fluxes of $\mathrm{HI}, \mathrm{CO}$, and $\mathrm{H} \alpha$, and a high ratio between rotational and dispersion velocities. The second one $(G B 1)$ has relatively red galaxies (high $U-B$ ), with an increasing central mass concentration and a decreasing central $\mathrm{M} / \mathrm{L}$, while the ratio $\mathrm{MHI} / \mathrm{B}$ luminosity is low. The third one $(G B 2)$ has galaxies with low and decreasing $V$ luminosity, an increasing central $\mathrm{M} / \mathrm{L}$, a decreasing $\mathrm{MHI} / \mathrm{B}$ luminosity and a low $\mathrm{Fe} / \mathrm{H}$. In summary, the group $G A 2$ is rather active and massive, $G B 1$ accretes gas toward its centre and increases its global $V$ luminosity, and $G B 2$ looks like a dead end with gas-poor galaxies and decreasing $V$ luminosity. These are three different evolutionary paths grouping objects with similar histories that models will have to explain.

Since it is based on observational data, the evolutionary scenario depicted by the tree is globally consistent with current thoughts about dwarf history and physics (e.g. Mateo 1998; Carrera et al. 2002; Wilcots \& Miller 1998; Gallagher \& Wyse 1994). It has the advantage of being built without choosing any particular parameters and is the unique way to synthesise the information contained in such a multivariate problem. In addition, astrocladistics introduces the evolution in the analysis itself, providing a direct view of the diversification process.

The results are based on the data and naturally depend on their quality, in particular on distance estimations of galaxies. It is not expected that error bars in the original data bring much noise in the result because initial quantitative data are binned (coded). In the same manner, the effect of any aberrant point is smoothed out in the analysis since the result is a synthesis of all the input information. In any case, a cladistics analysis has this invaluable quality of being entirely transparent and falsifiable, so that it is always possible to modify original values, to modify the way the coding is done, to introduce weights to characters, and to compare the resulting phylogenies.

\section{Conclusion}

We conclude that branching evolution is the dominant diversification process among the 36 dwarf galaxies of the Local Group since the diversity is organised in a tree-like or hierarchical way. Phylogenetic systematics is thus applicable to astrophysics and provides a powerful tool for understanding galaxy formation and evolution. The tree depicts relationships between galaxies by taking physical and chemical character changes into account. It thus provides an opportunity to formulate new inferences on galaxy evolution by providing a synthetic view of current knowledge.

We have identified at least three different evolutionary groups, each one with its own characteristics and own evolution. One is composed of massive and star-forming galaxies, another has galaxies that seem to increase the luminosity while transferring mass toward their centre, and the last one looks like the dead end of dwarf galaxy evolution with dimming and concentrating objects.

Morphology classification appears to be too simplistic for understanding the evolution of dwarf galaxies. From the trees we have found, we derive that some kinds of dIrrs are progenitors of both dSphs and other kinds of dIrrs. Two of our evolutionary groups are composed of dSphs, the other one of dIrrs, and the intermediate type is spread over the tree.

We must stress that the phylogeny we found is entirely based on the data input. The best way to avoid introducing a subjective bias is to consider all available characters excluding obviously redundant ones, as is done in phylogenetic systematics. As new data and/or new characters become available and introduced into the analysis, the current phylogeny might change somewhat in the future. This is the normal progress of knowledge. In the particular case of the Dwarf galaxies of the Local Group, spectrophotometric observations will certainly be invaluable for astrocladistics, but this would ideally require that homogeneous data on a significant sample be available.

Extension of this study to many more types of galaxies in the Universe is in progress and will possibly yield the basis for a new taxonomy of galaxies.

Acknowledgements. We thank the anonymous referee for suggestions that lead to a big improvement in the paper. Most computations of the results presented in this paper were performed within the CIMENT project in Grenoble. E.J.P.D. was supported by the Genopole Montpellier Languedoc-Roussillon and the Action Bioinformatique inter-EPST of the CNRS, and this work represents the contribution No.-2006-030 of the Institut des Sciences de 1'Evolution de Montpellier (UMR 5554 - CNRS).

\section{References}

Bremer, K. 1994, Cladistics, 10, 295

Carrera, R., Aparicio, A., Martinez-Delgado, D., \& Alonso-Garcia, J. 2002, AJ, 123,3199

Darwin, C. 1859, The Origin of Species (London: John Murray)

de Vaucouleurs, G. 1959, Handbuch der Physik, 53, 275

Felsenstein, J. 1985, Evolution, 39, 783

Fitch, W. M. 1971, Systematic Zoology, 20, 406

Fraix-Burnet, D. 2004, in Penetrating Bars through Masks of Cosmic Dust: The Hubble Tuning Fork strikes a New Note, ed. D. L. Block, I. Puerari, K. C. Freeman, R. Groess, \& E. K. Block (Dordrecht: Kluwer Academic Publishers), 301 [arXiv: astro-ph/0406512]

Fraix-Burnet, D., Choler, P., \& Douzery, E. J. P. 2003, Ap\&SS, 284, 535

Fraix-Burnet, D., Choler, P., Douzery, E. J. P., \& Verhamme, A. 2006a, Journal of Classification, in press [arXiv: astro-ph/0602581]

Fraix-Burnet, D., Douzery, E. J. P., Choler, P., \& Verhamme, A. 2006b, Journal of Classification, in press [arXiv: astro-ph/0602580]

Gallagher, J. S., \& Wyse, R. F. G. 1994, PASP, 106, 1225 
Grebel, E. K. 2005, in Near-Field Cosmology With Dwarf Elliptical Galaxies Proc. IAU Coll. No. 198, ed. H. Jerjen, \& B. Binggeli, 1 [arXiv: astro-ph/0509150]

Hennig, W. 1965, Annual Review of Entomology, 10, 97

Hubble, E. P. 1922, ApJ, 56, 162

Hubble, E. P. 1926, ApJ, 64, 321

Hubble, E. P. 1936, The Realm of Nebulae (New Haven: Yale Univ. Press)

Kormendy, J., \& Bender, R. 1996, ApJ, 464, L119

Mateo, M. 1998, ARA\&A, 36, 435

Maddison, W. P., \& Maddison, D. R. 2004, Mesquite: a modular system for evolutionary analysis, version 1.05 (http://mesquiteproject.org)

Mighell, K. J., \& Burke, C. J. 1999, AJ, 118, 366

Moore, B., Ghigna, S., Governato, F., et al. 1999, ApJ, 524, L19

Ricotti, M., \& Gnedin, N. Y. 2005, ApJ, 629, 259

Roberts, M. S., \& Haynes, M. P. 1994, ARA\&A, 32, 115

Robinson, P. M. W., \& Robert, J. O. 1996, Research in Humanities Computing, 4,115

Sandage, A. 1961, The Hubble Atlas of Galaxies (Washington: Carnegie Inst. Washington)
Sauvage, M., Stasinska, G., \& Schaerer, D., ed. 2002, The Evolution of Galaxies. II- Basic Building Blocks (Kluwer Academic Publishers)

Swofford, D. L. 1998, PAUP*. Phylogenetic Analysis Using Parsimony (and Other Methods), Version 4, (Sinauer Associates, Sunderland, Massachusetts)

van den Bergh, S. 2000, PASP, 112, 529

Venn, K. A., Irwin, M., Shetrone, M. D., Tout, C. A., Hill, V., \& Tolstoy, E. 2004, AJ, 128, 1177

Vilchez, J., Stasinska, G., \& Perez, E., ed. 2001, The Evolution of Galaxies. I- Observational Clues (Kluwer Academic Publishers)

Watanabe, M., Kodaira, K., \& Okamura, S. 1985, ApJ, 292, 72

Wells, R. S. 1987, in Biological Metaphor and Cladistic Classification: An Interdisciplinary Perspective, ed. H. M. Hoenigswald, \& L. F. Wiener (Philadelphia), 39

Whitmore, B. C. 1984, ApJ, 278, 61

Wilcots, E. M., \& Miller, B. W. 1998, AJ, 116, 2363

Wiley, E. O., Siegel-Causey, D., Brooks, D. R., \& Funk, V. A. 1991, The Compleat Cladist. A Primer of Phylogenetic Procedures, The University of Kansas, Museum of Natural History) special publication No. 19 
D. Fraix-Burnet et al.: Towards a phylogenetic analysis of galaxy evolution, Online Material $p 1$

\section{Online Material}


D. Fraix-Burnet et al.: Towards a phylogenetic analysis of galaxy evolution, Online Material $p 2$
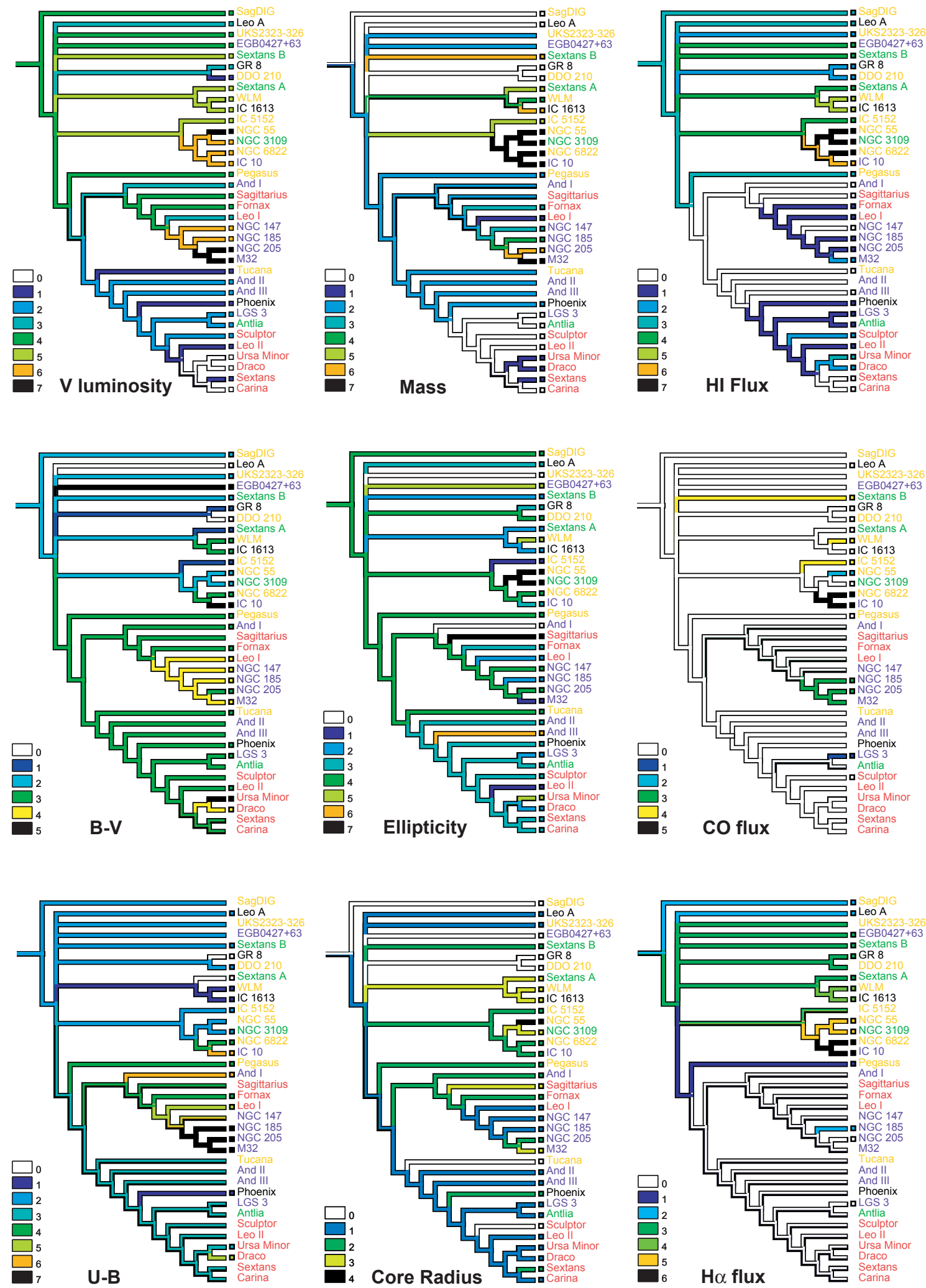

Fig. 3. Colour-coded projection of characters with the convention that the white code is always the lowest value of the corresponding parameter. Small squares at the leaves (before galaxy name) indicate a documented value for that character. The other leaves are given a value deduced from a parsimony analysis, providing a prediction for the corresponding galaxy. The colour code for galaxy names is given at the end of figure. Morphology is plotted but was not used to derive the tree. 
D. Fraix-Burnet et al.: Towards a phylogenetic analysis of galaxy evolution, Online Material $p 3$
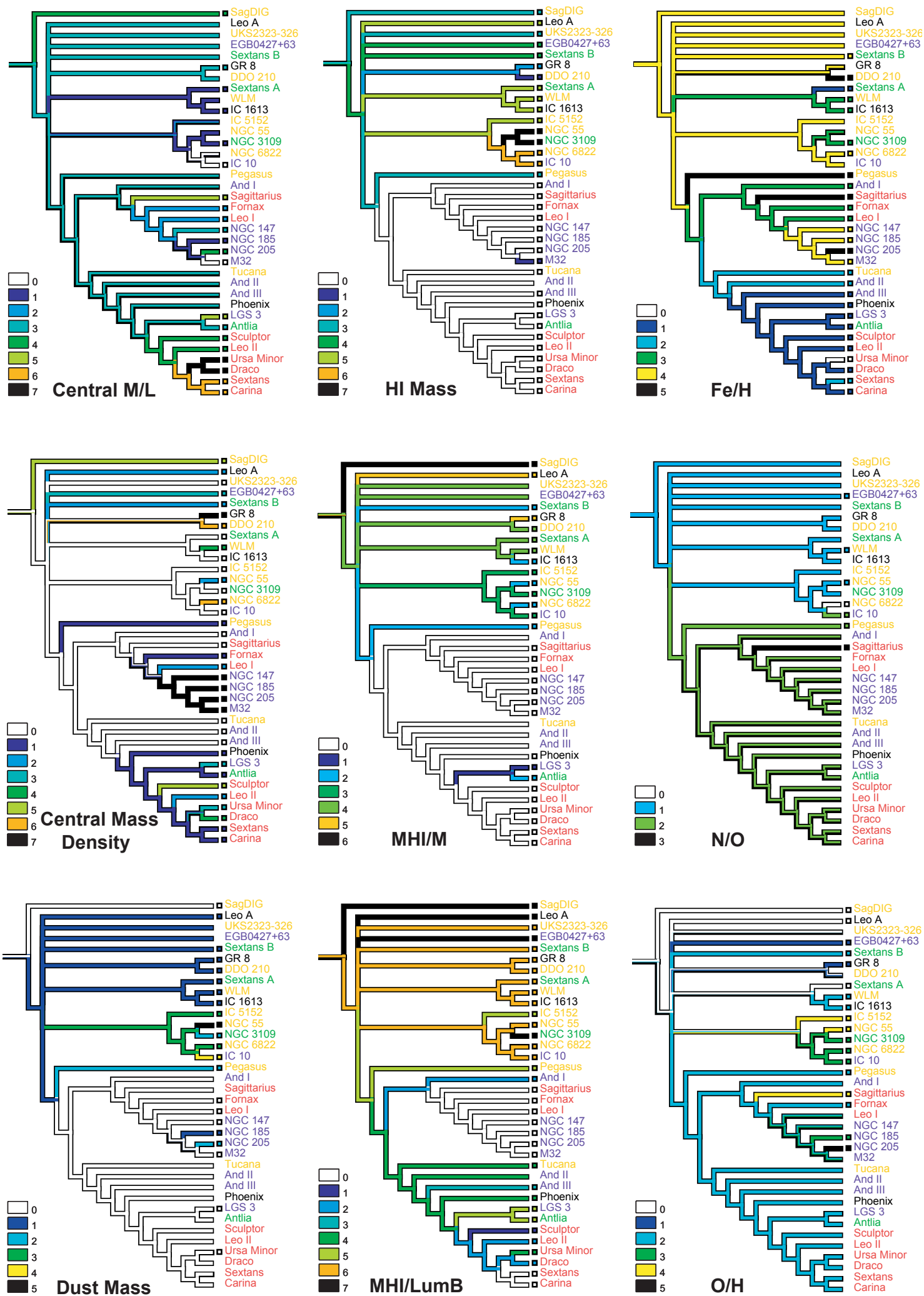

Fig. 3. continued. 
D. Fraix-Burnet et al.: Towards a phylogenetic analysis of galaxy evolution, Online Material p 4
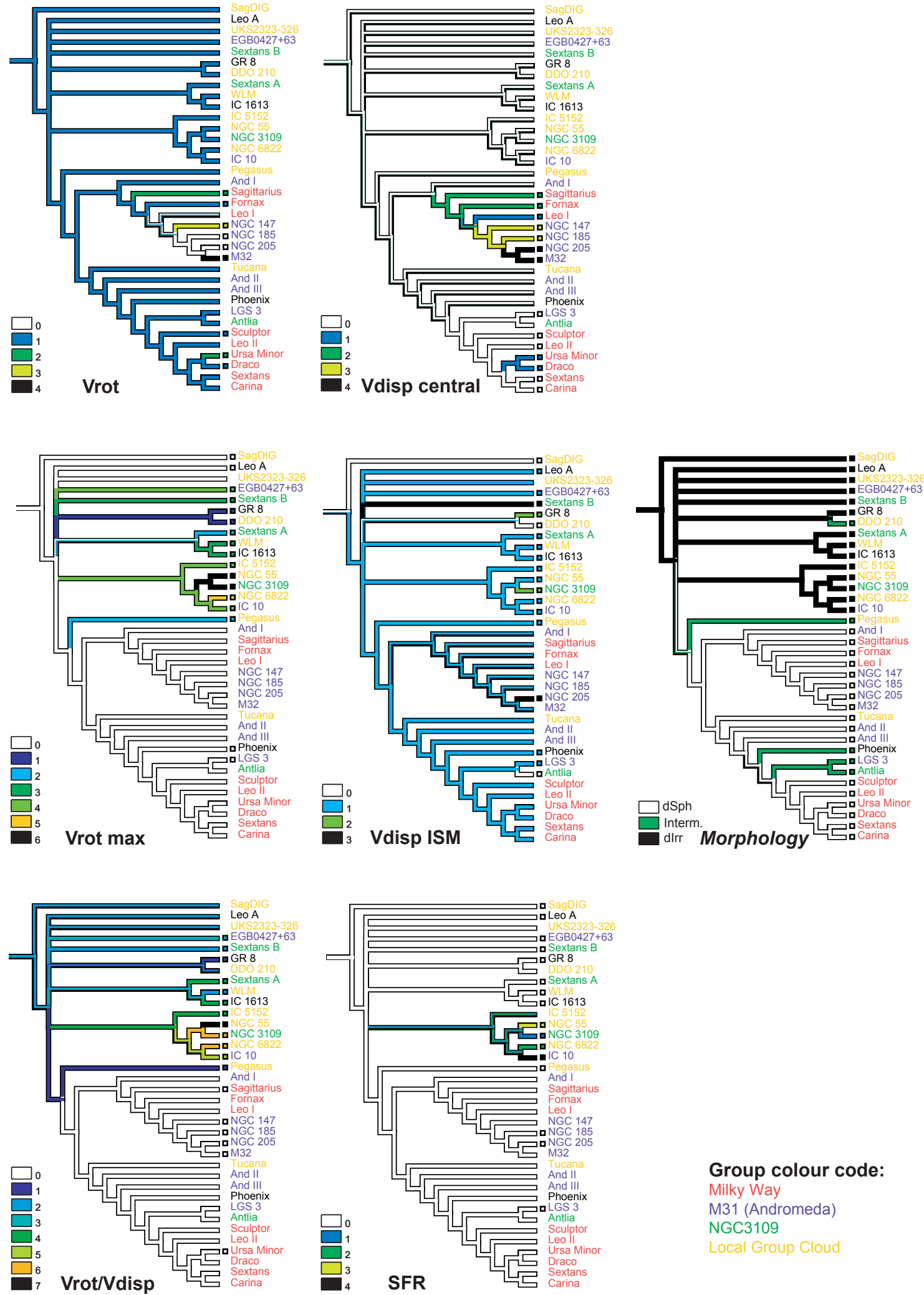

Group colour code:

Milky Way

M31 (Andromeda)

NGC3109

-ocal Group Cloud

Fig. 3. continued. 